ARTICLE

DOI: $10.1038 /$ s41467-018-05909-8

\title{
Color-stable highly luminescent sky-blue perovskite light-emitting diodes
}

\author{
Jun Xing (1) 1,2,7, Yongbiao Zhao ${ }^{3}$, Mikhail Askerka², Li Na Quan (1) 2, Xiwen Gong (1) 2, Weijie Zhao ${ }^{1}$, Jiaxin Zhao', \\ Hairen Tan (1) 2, Guankui Long (1) 2, Liang Gao ${ }^{2,4}$, Zhenyu Yang (1) 2, Oleksandr Voznyy (1) 2, Jiang Tang (1) 4, \\ Zheng-Hong Lu (i] ${ }^{3}$, Qihua Xiong ${ }^{1,5,6} \&$ Edward H. Sargent (i) ${ }^{2}$
}

Perovskite light-emitting diodes (PeLEDs) have shown excellent performance in the green and near-infrared spectral regions, with high color purity, efficiency, and brightness. In order to shift the emission wavelength to the blue, compositional engineering (anion mixing) and quantum-confinement engineering (reduced-dimensionality) have been employed. Unfortunately, LED emission profiles shift with increasing driving voltages due to either phase separation or the coexistence of multiple crystal domains. Here we report color-stable skyblue PeLEDs achieved by enhancing the phase monodispersity of quasi-2D perovskite thin films. We selected cation combinations that modulate the crystallization and layer thickness distribution of the domains. The perovskite films show a record photoluminescence quantum yield of $88 \%$ at $477 \mathrm{~nm}$. The corresponding PeLEDs exhibit stable sky-blue emission under high operation voltages. A maximum luminance of $2480 \mathrm{~cd} \mathrm{~m}^{-2}$ at $490 \mathrm{~nm}$ is achieved, fully one order of magnitude higher than the previous record for quasi-2D blue PeLEDs.

\footnotetext{
${ }^{1}$ Division of Physics and Applied Physics, School of Physical and Mathematical Sciences, Nanyang Technological University, Singapore 637371, Singapore.

${ }^{2}$ Department of Electrical and Computer Engineering, University of Toronto, 10 King's College Road, Toronto, ON M5S 3G4, Canada. ${ }^{3}$ Department of Materials Science and Engineering, University of Toronto, 184 College Street, Toronto, ON M5S 3E4, Canada. ${ }^{4}$ Wuhan National Laboratory for Optoelectronics (WNLO) and School of Optical and Electronic Information, Huazhong University of Science and Technology (HUST), 430074 Wuhan, China. ${ }^{5}$ NOVITAS, Nanoelectronics Centre of Excellence, School of Electrical and Electronic Engineering, Nanyang Technological University, Singapore 639798, Singapore. ${ }^{6}$ MajuLab, CNRS-UNS-NUS-NTU International Joint Research Unit, UMI-3654, Singapore 639798, Singapore. ${ }^{7}$ Present address: Shaanxi Institute of Flexible Electronics (SIFE), Northwestern Polytechnical University (NPU), 127 West Youyi Road, 710072 Xi'an, Shaanxi, China. These authors contributed equally: Jun Xing, Yongbiao Zhao. Correspondence and requests for materials should be addressed to Z.-H.L. (email: zhenghong.lu@utoronto.ca) or to Q.X. (email: Qihua@ntu.edu.sg) or to E.H.S. (email: ted.sargent@utoronto.ca)
} 
M etal halide perovskites are emerging as a promising candidate for solution-processed optoelectronics, including photovoltaics, photodetectors, lasers and light-emitting diodes (LEDs) $)^{1-18}$. Thanks to their excellent properties such as a tunable bandgap, high photoluminescence quantum yield (PLQY), and high color purity, perovskite LEDs (PeLEDs) have attracted intense research interest. Recently, green- and near-infrared-emitting PeLEDs reached impressive external quantum efficiencies (EQEs) exceeding 10\%,19-21. However, producing color-stable and efficient blue PeLEDs required for lighting and displays remains challenging, especially in light of limited materials stabilty $22-25$.

Conventional metal halide perovskites exhibit a threedimensional (3D) structure with a chemical formula of $\mathrm{AMX}_{3}$ $\left(\mathrm{A}=\mathrm{CH}_{3} \mathrm{NH}_{3}\right.$ (MA), $\left.\mathrm{Cs} ; \mathrm{M}=\mathrm{Pb}, \mathrm{Sn} ; \mathrm{X}=\mathrm{Cl}, \mathrm{Br}, \mathrm{I}\right)$. The bandgap of the mixed halide perovskites can be tuned monotonically by controlling the halogen component. One widely employed strategy to achieve blue PeLEDs (emission wavelength between 450 and $490 \mathrm{~nm}$ ) is therefore to employ $\mathrm{Cl}: \mathrm{Br}$-mixed halide perovskites as the emissive material. However, the migration of the halogen ions enables phase segregation into $\mathrm{Cl}$-rich and $\mathrm{Br}$-rich phases under illumination and voltage bias ${ }^{24,26,27}$. As a result, the electroluminescence (EL) wavelength undesirably shifts from blue to green during device operation.

Quasi-two-dimensional (quasi-2D) perovskites provide another pathway to achieve blue PeLEDs. Their reduced-dimensional structure has a standard formula of $\mathrm{B}_{2} \mathrm{~A}_{n-1} \mathrm{M}_{n} \mathrm{X}_{3 n+1}$, where $\mathrm{B}$ is a long-chain ligand such as butylammonium (BA) or phenylethylammonium (PEA) (inset of Fig. 1a). By reducing the number of inorganic layers $(n)$, one may tune the bandgap of quasi-2D perovskites from ca. $2.6 \mathrm{eV}(n=4)$, to $2.7 \mathrm{eV}(n=3), 2.9 \mathrm{eV}(n=$ 2), and $3.1 \mathrm{eV}(n=1)$ (Fig. 1a) ${ }^{28}$. Quasi-2D blue PeLEDs have been also reported; however, they exhibit poor color stability under LEDs operation ${ }^{23,29-32}$. This is due to the presence of multiple emission peaks arising from mixed phases that exhibit inefficient charge transfer and different emission features dependent on bias voltage.

So far, PEA-based quasi-2D perovskites exhibited a welldefined single emission peak as a result of efficient charge transfer between quantum wells with various $n$ values $8,28,33$. However, PEA-based quasi-2D perovskites that use only $\mathrm{Br}$ could not reach blue emission due to the presence of the phase with large $n^{28,33}$. We postulate that the formation of different phases within a perovskite film could arise due to the imbalance of crystallization of different phases. Specifically, strong van der Waals interaction between the stacked bulky organic cations ${ }^{8,28}$ makes the formation of $2 \mathrm{D}$ perovskite with $n=1$ most favorable (Supplementary
Note 1 and Supplementary Fig. 1). This consumes a major portion of bulk organic cations and leaves the short-chain precursors (those associated with the repeat unit of 3D crystals) to nucleate phases with high $n$. As a result, a mixed phase that includes high$n$ components shows green emission due to efficient carrier funneling to the smallest-bandgap phase.

In this report, we therefore aim at slowing the formation of the pure $n=1$ phase to improve the monodispersity of $n=2,3,4$ phases enabling more controlled formation of a quasi-2D $\mathrm{PEA}_{2} \mathrm{~A}_{n-1} \mathrm{~Pb}_{n} \mathrm{X}_{3 n+1}$ perovskite with a desired $n$. We achieve this by replacing long ligands (PEA) with shorter ones (iso-propylammonium, IPA), an improvement that reduces van der Waals interactions, therefore destabilizing the $n=1$ phase. Slower crystallization of $n=1$ enhances purity and monodispersity of $n=2,3$, and 4 phases. The as-synthesized perovskite films exhibit a single emission peak and color-stable blue emission $(477 \mathrm{~nm})$ with a record PLQY of $88 \%$. The PL wavelength exhibits no shift under 325-nm irradiation $\left(7 \mathrm{~W} \mathrm{~cm}^{-2}\right)$ for $1 \mathrm{~h}$. We further fabricate sky-blue PeLEDs with a maximum luminance of $2480 \mathrm{~cd} \mathrm{~m}^{-2}$ at the wavelength of $490 \mathrm{~nm}$, a luminance that is one order of magnitude higher than the previous record for quasi-2D blue PeLEDs (Supplementary Table 1) 23,29-32.

\section{Results}

Synthesis and structural analysis of perovskite films. We synthesized blue emission perovskites by mixing $\mathrm{PbBr}_{2}, \mathrm{MABr}, \mathrm{CsBr}$, $\mathrm{PEABr}$, and IPABr in the solution of dimethyl sulfoxide (DMSO) and using a one-step spin-coating method (see Methods). The mixture of MA and Cs cations has been shown previously to improve the crystallinity and stability of perovskite films $\mathrm{s}^{34}$. The ratio of $\mathrm{MABr} / \mathrm{CsBr}$ to $\mathrm{PEABr}$ and $\mathrm{PbBr}_{2}$ in the precursor solution was kept constant at 3:4:5, targeting an average perovskite formula $\mathrm{PEA}_{2} \mathrm{~A}_{1.5} \mathrm{~Pb}_{2.5} \mathrm{Br}_{8.5}(\mathrm{~A}=\mathrm{MA}$ and $\mathrm{Cs})$. We explored a number of additives to tune the emission properties: $\mathrm{IPABr}$, ethylammonium bromide (EABr), $n$-propylammonium bromide $(\mathrm{PABr})$, and butylammonium bromide (BABr). We found that only the addition of $\mathrm{IPABr}$ resulted in a perovskite product with high PLQY.

Density functional theory (DFT) simulations (Fig. 1b) show that when PEABr and IPABr ligands are used in combination, the formation energy of the 2D perovskite with $n=1$ changes from -7.2 (more stable) to $-6.5 \mathrm{eV}$ (less stable), indicating a partial destabilization of $n=1$ phase compared to the $n=2,3$, and 4 phases. Due to stoichiometry constraints, we obtained only quasi$2 \mathrm{D}$ perovskites with low number of inorganic layers, which is critical to achieving blue emission.
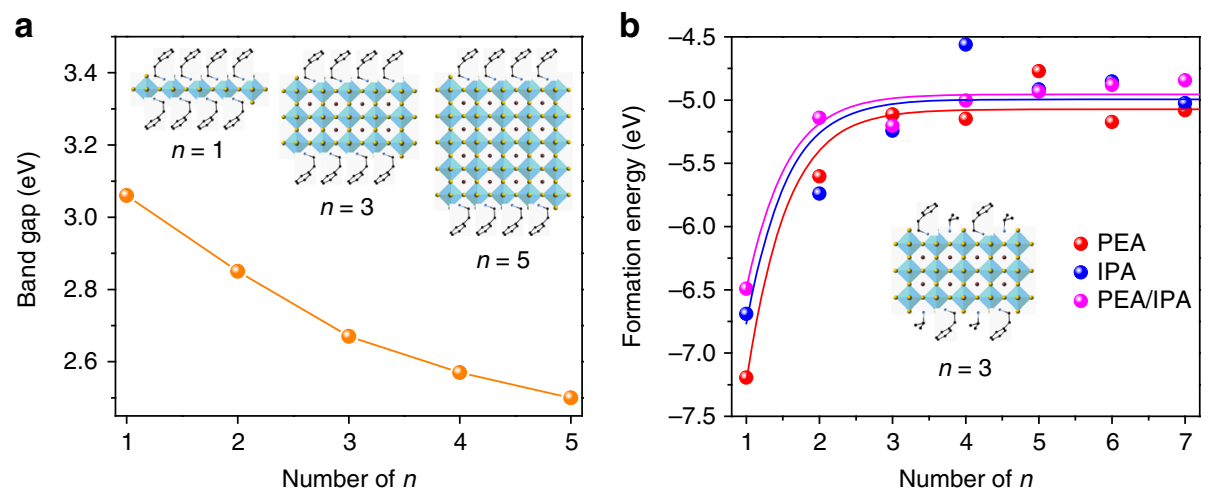

Fig. 1 Bandgap and formation energy calculations of quasi-2D perovskite. a Bandgap of quasi-2D perovskite with different number of layers, $n$. These values are determined by TA measurement. The inset is the atomic model of the quasi-2D perovskite with $n=1,3$, and 5. $\mathbf{b}$ Calculated formation energy of PEABr, $\mathrm{IPABr}$, and their mixed quasi-2D perovskites with different $n$ value. The inset is an atomic model of the PEA/IPA mixed quasi-2D perovskite with $n=3$ 
Table 1 Summary of the composition of the perovskite samples and their corresponding PL peaks and PLQY

\begin{tabular}{|c|c|c|c|c|c|c|}
\hline & $\mathrm{CsPbBr}_{3}$ & $\mathrm{MAPbBr}_{3}$ & $\mathrm{PEA}_{2} \mathrm{PbBr}_{4}$ & IPABr & PL peak $(\mathrm{nm})$ & PLQY\% \\
\hline Sample I & 0.5 & 0.1 & 0.4 & 0 & 504 & 69 \\
\hline Sample II & 0.5 & 0.1 & 0.4 & 0.1 & 498 & 79 \\
\hline Sample IV & 0.5 & 0.1 & 0.4 & 0.4 & 480 & 73 \\
\hline Sample V & 0.45 & 0.15 & 0.4 & 0.4 & 477 & 88 \\
\hline Sample VI & 0.4 & 0.2 & 0.4 & 0.4 & 473 & 82 \\
\hline Sample VII & 0.5 & 0.1 & 0.4 & 0.6 & 467 & 53 \\
\hline
\end{tabular}

a
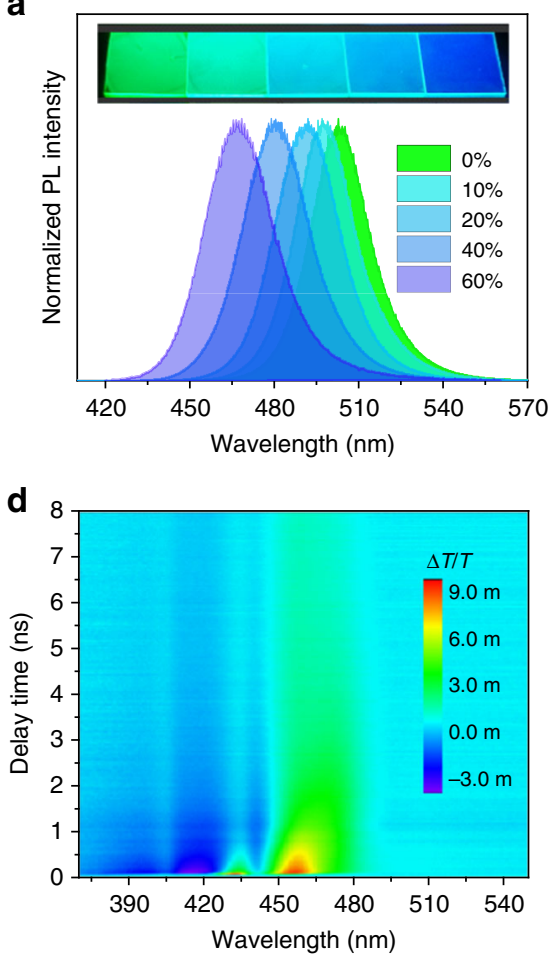

b

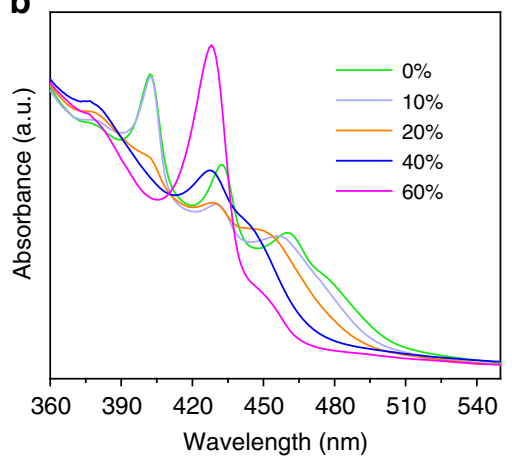

e

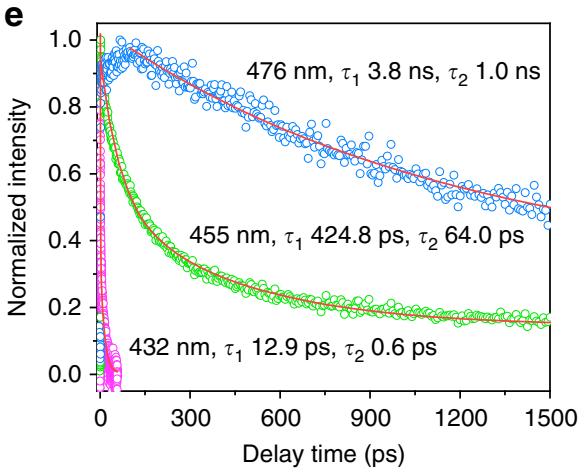

C
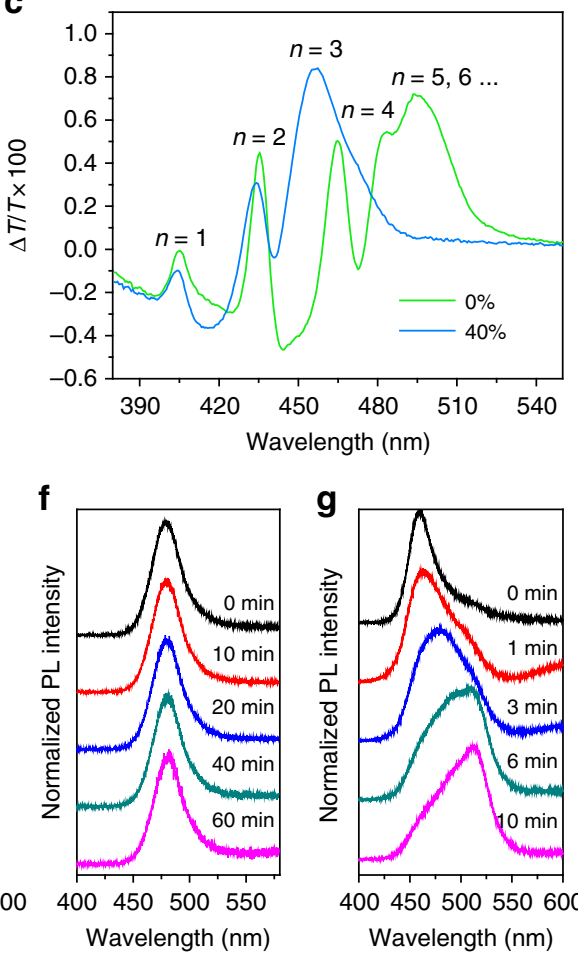

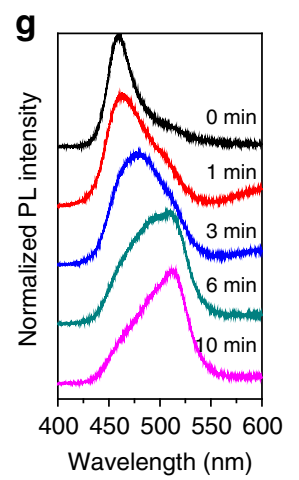

Fig. 2 Photophysical properties of quasi-2D perovskite. a PL and $\mathbf{b}$ absorption spectra of perovskite $\mathrm{PEA}_{2} \mathrm{~A}_{1.5} \mathrm{~Pb}_{2.5} \mathrm{Br}_{8.5}$ with $0-60 \%$ IPABr additive. The inset is a photograph of the corresponding films under $365 \mathrm{~nm}$ UV irradiation. c TA spectra of $\mathrm{PEA}_{2} \mathrm{~A}_{1.5} \mathrm{~Pb}_{2.5} \mathrm{Br}_{8.5}$ with 0 and $40 \%$ IPABr. d, e TA time delay of $\mathrm{PEA}_{2} \mathrm{~A}_{1.5} \mathrm{~Pb}_{2.5} \mathrm{Br}_{8.5}$ with $40 \%$ IPABr. PL spectra of quasi-2D perovskite (f) and $\mathrm{MAPbCl}_{1.5} \mathrm{Br}_{1.5}(\mathbf{g})$ film under continuous laser radiation (325 nm, $7 \mathrm{~W} \mathrm{~cm}^{-2}$ ) for different exposure times

$\mathrm{X}$-ray diffraction (XRD) results reveal the layered structure of formed perovskite thin films (Supplementary Fig. 2). Diffraction peaks at $4.35,8.74,13.5,27.2$, and 31.55 degrees correspond to the $\left(\begin{array}{lll}0 & 0 & l\end{array}\right)$ series of Bragg reflections of the quasi-2D perovskite. The lowest angle of $4.35^{\circ}$ corresponds to the lattice fringe of $2.0 \mathrm{~nm}$, which we assign to the interlayer spacing in the quasi-2D perovskite phase with $n=2^{35}$.

Optical properties of perovskite films. Figure 2a shows PL spectra of the perovskite films. Pristine $\mathrm{PEA}_{2} \mathrm{~A}_{1.5} \mathrm{~Pb}_{2.5} \mathrm{Br}_{8.5}$ perovskite emits at $504 \mathrm{~nm}$; the addition of IPABr progressively shifts the PL peak from 497 to $467 \mathrm{~nm}$ as the $\mathrm{IPABr} / \mathrm{Pb}$ ratio increases from 10 to $60 \%$. The corresponding photograph of the perovskite films under UV light is shown in the inset of Fig. 2a. The highest PLQY of $73 \%$ was obtained with an IPABr ratio of $40 \%$ (Table 1). By tuning the fractions of $\mathrm{MABr}$ and $\mathrm{CsBr}$, we further optimized the PLQY and obtained a highest value of $88 \%$ with the PL peak at $477 \mathrm{~nm}$.
UV-Vis absorption spectra were used to characterize the optical bandgap and excitonic features of these perovskites. In Fig. 2b, multiple excitonic absorption features at 3.1, 2.9, 2.7, and $2.6 \mathrm{eV}$ correspond to the perovskite phases with $n=1,2,3$, and 4, respectively ${ }^{28}$. We also observed a blueshift of the absorption edge of the perovskite films with increased $\mathrm{IPABr}$ fraction. We attribute this blueshift to the fact that the component of lowest- $n$ and highest- $n$ phases was inhibited gradually, while the intermediate $n$ phase $(n=2,3,4)$ grew faster instead (Fig. $2 \mathrm{~b})$.

Transient absorption spectroscopy. To gain further insights into the dynamics of photocarriers in the bright blue quasi-2D perovskites, we carried out transient absorption (TA) studies (Fig. 2c). In the control sample (without IPABr), we observed five distinct bleach peaks at 405, 435, 465, 483, and $495 \mathrm{~nm}$. The peak locations of these transitions are in good agreement with peaks in the steady-state absorption spectra (Fig. 2b). These results show that the quasi-2D perovskite $\mathrm{PEA}_{2} \mathrm{~A}_{1.5} \mathrm{~Pb}_{2.5} \mathrm{Br}_{8.5}$ films are not 
a

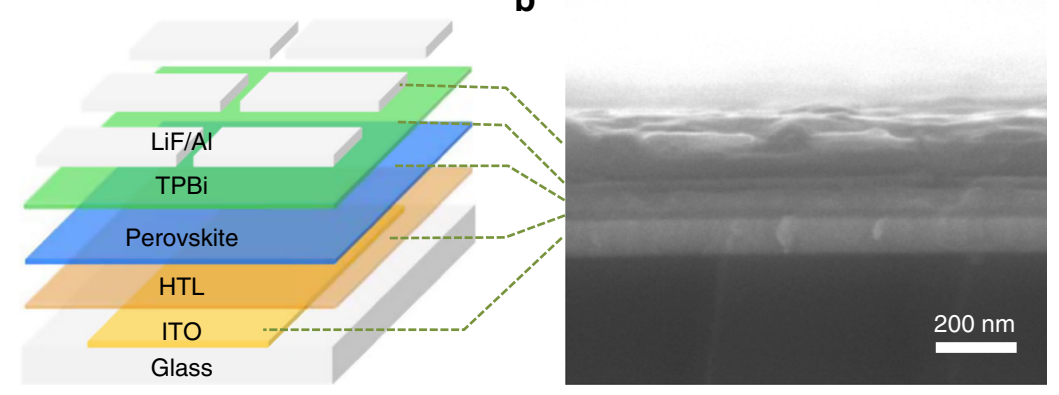

C

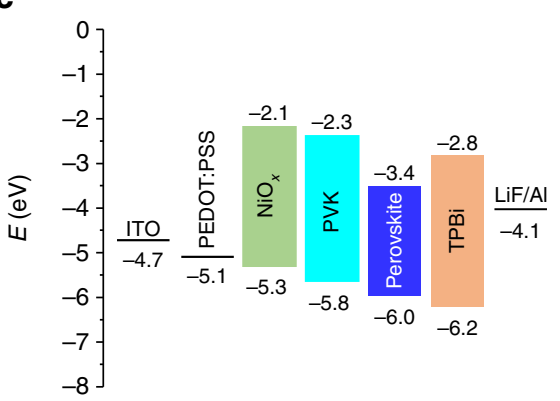

d

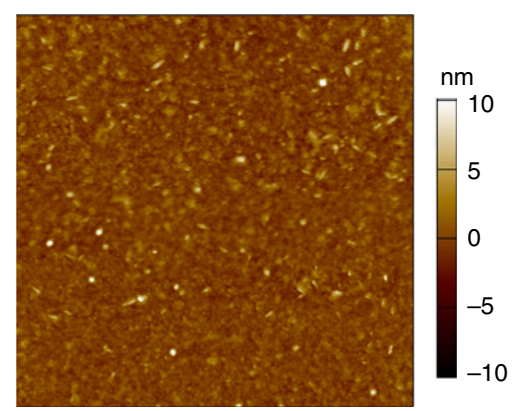

Fig. 3 Device structure of PeLEDs. a Device structure, $\mathbf{b}$ cross-sectional SEM image and c energy level alignment of the PeLEDs, showing conduction and valence-band levels with respect to vacuum. The energy level of perovskite layer was determined by UPS measurement. $\mathbf{d}$ AFM image of perovskite film on PEDOT:PSS. Scan area is $5 \times 5 \mu \mathrm{m}^{2}$. The surface roughness was measured to be around $1.0 \mathrm{~nm}$

single-phase, but instead consist of a mixture of phases with $n=$ $1,2,3,4,5$, and higher ${ }^{28}$. The band edge energies of the perovskites with $n \geq 5$ are closely spaced and difficult to distinguish. Therefore, we propose that bulk perovskite phases with larger $n$ values may also exist in the $\mathrm{PEA}_{2} \mathrm{~A}_{1.5} \mathrm{~Pb}_{2.5} \mathrm{Br}_{8.5}$ films. In contrast, in the sample with $40 \%$ IPABr additive, only four distinctive phases with $n=1,2,3$, and 4 were observed with no spectral or XRD features that are normally attributed to $n \geq 5$.

We then investigated the decay kinetics of multi-exciton bleaching (Fig. 2d). We extracted three distinct kinetics at 432, 455 , and $476 \mathrm{~nm}$ corresponding to $n=2,3$, and 4 , that revealed the following time constants after fitting (Fig. 2e):

$13 \pm 0.1 \mathrm{ps}$ and $0.6 \pm 0.01 \mathrm{ps}(432 \mathrm{~nm})$;

$420 \pm 10$ ps and $64 \pm 2$ ps $(455 \mathrm{~nm})$; and

$4 \pm 1 \mathrm{~ns}$ and $1.0 \pm 0.1 \mathrm{~ns}(476 \mathrm{~nm})$

In light of previous reports of very fast charge transfer among perovskite phases ${ }^{36}$, we assign the fast component at $432,455 \mathrm{~nm}$ $(n=2,3)$ to the charge transfer to perovskite with $n=4$ and ascribe the slow component to charge trapping ${ }^{28}$. We attribute the fast and the slow features at $476 \mathrm{~nm}$ (emitting domain, $n=4$ ) to a nonradiative and a radiative process, respectively. Notably, at $476 \mathrm{~nm}$, the timescale of the rise of the bleach intensity $\Delta T / T$ (during the first $100 \mathrm{ps}$ ) is in good agreement with the charge transfer (reflected by lifetime decay) that was previously reported for a perovskite phase with $n=3^{36}$.

We further studied the color stability of these improved quasi$2 \mathrm{D}$ perovskites. The $\mathrm{PL}$ spectra of the $\mathrm{PEA}_{2} \mathrm{~A}_{1.5} \mathrm{~Pb}_{2.5} \mathrm{Br}_{8.5}$ perovskite with $40 \% \mathrm{IPABr}$ under continuous $325 \mathrm{~nm}$ laser radiation $\left(7 \mathrm{~W} \mathrm{~cm}^{-2}\right)$ are shown in Fig. $2 \mathrm{f}$. The PL maximum is unchanged even after irradiation for $60 \mathrm{~min}$. In contrast, the PL peak of a mixed halide perovskite $\mathrm{MAPbBr}_{1.5} \mathrm{Cl}_{1.5}$ film shifts from 460 to $515 \mathrm{~nm}$ within $10 \mathrm{~min}$ of illumination under the same conditions (Fig. 2g).

Device structure and performance. Encouraged by the above results, we further fabricated PeLEDs using perovskite films with highest PLQYs (Sample V, see Methods for details). As indicated in Fig. 3a, b, we began with the following PeLED architecture: ITO/PEDOT:PSS $(30 \mathrm{~nm}) /$ perovskite film $\quad(40-125 \mathrm{~nm}) / \mathrm{TPBi}$ $(50 \mathrm{~nm}) / \mathrm{LiF}(1 \mathrm{~nm}) / \mathrm{Al}(100 \mathrm{~nm})$. We measured the energy levels of the perovskites by using ultraviolet photoelectron spectroscopy (UPS, Supplementary Fig. 3). As shown in the atomic force microscopy (AFM, Fig. 3d) image, the surface roughness of the perovskite film on PEDOT:PSS is only $1 \mathrm{~nm}$, which is sufficiently small to avoid current leakage.

Compared to $3 \mathrm{D}$ perovskites, quasi-2D perovskites exhibit lower conductivity and thus their LED architecture usually requires thinner active layers. To explore the effect of the thickness on our device performance, we studied the PeLEDs with thicknesses of the active layer ranging from $125 \mathrm{~nm}$ (Device I) to $80 \mathrm{~nm}$ (Device II), $60 \mathrm{~nm}$ (Device III), and $40 \mathrm{~nm}$ (Device IV) (Supplementary Fig. 4). We found that devices with thinner active layers showed both increased current density and luminance (Fig. 4a). We also found that higher brightness $\left(2480 \mathrm{~cd} \mathrm{~m}^{-2}\right.$, Device IV, Fig. 4b) was achieved through a systematic reduction of thickness. The best EQEs, power efficiency, and current efficiency of $1.5 \%, 0.92 \mathrm{~lm} \mathrm{~W}^{-1}$, and $2.8 \mathrm{~cd} \mathrm{~A}^{-1}$, respectively, were found in Device II (Fig. $4 c-e$ ). We propose that a higher EQE can be achieved through improvement of the electron (ETLs) and hole (HTLs) transport layers to optimize the charge carrier injection and blocking functions. As indicated in Fig. 4b, c, the maximum luminance and the maximum EQE were obtained from different devices. Luminance $(L)$ is proportional to the product of current density $(J)$ and efficiency (EQE), indicating that luminance is affected by both current density and efficiency. While thinner perovskite films achieve higher $J$, they do not always have higher EQE. EQE is governed by exciton and charge carrier dynamics. Most exciton quenching processes scale with charge carrier concentrations, making quenching processes most pronounced at high current densities. The reduction of perovskite thickness also increases the chance of charge carrier leakage. More exciton quenching and more charge carrier leakage will result in lower EQE. Therefore, increasing the maximum current density can compensate for some of these EQE losses; however, 

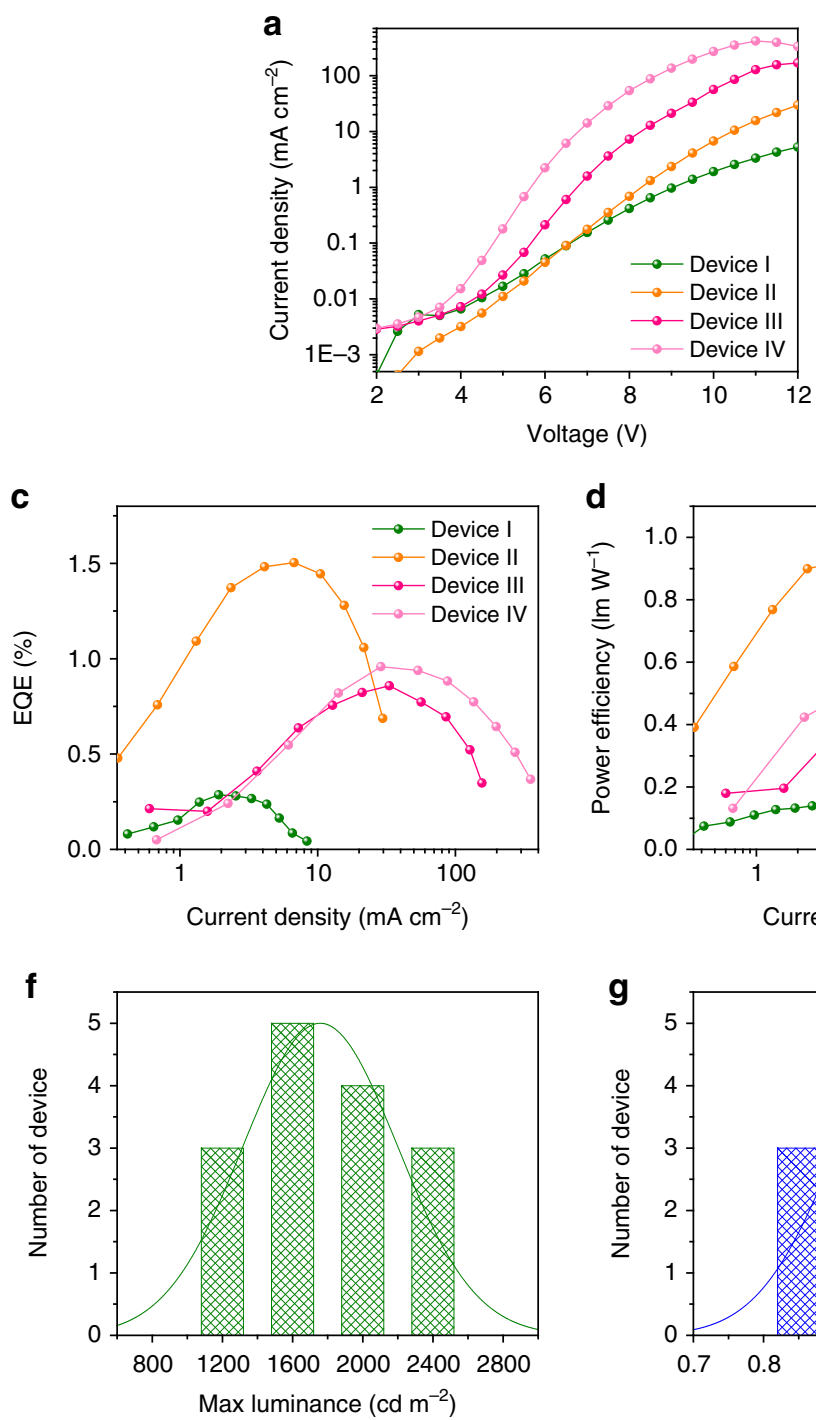
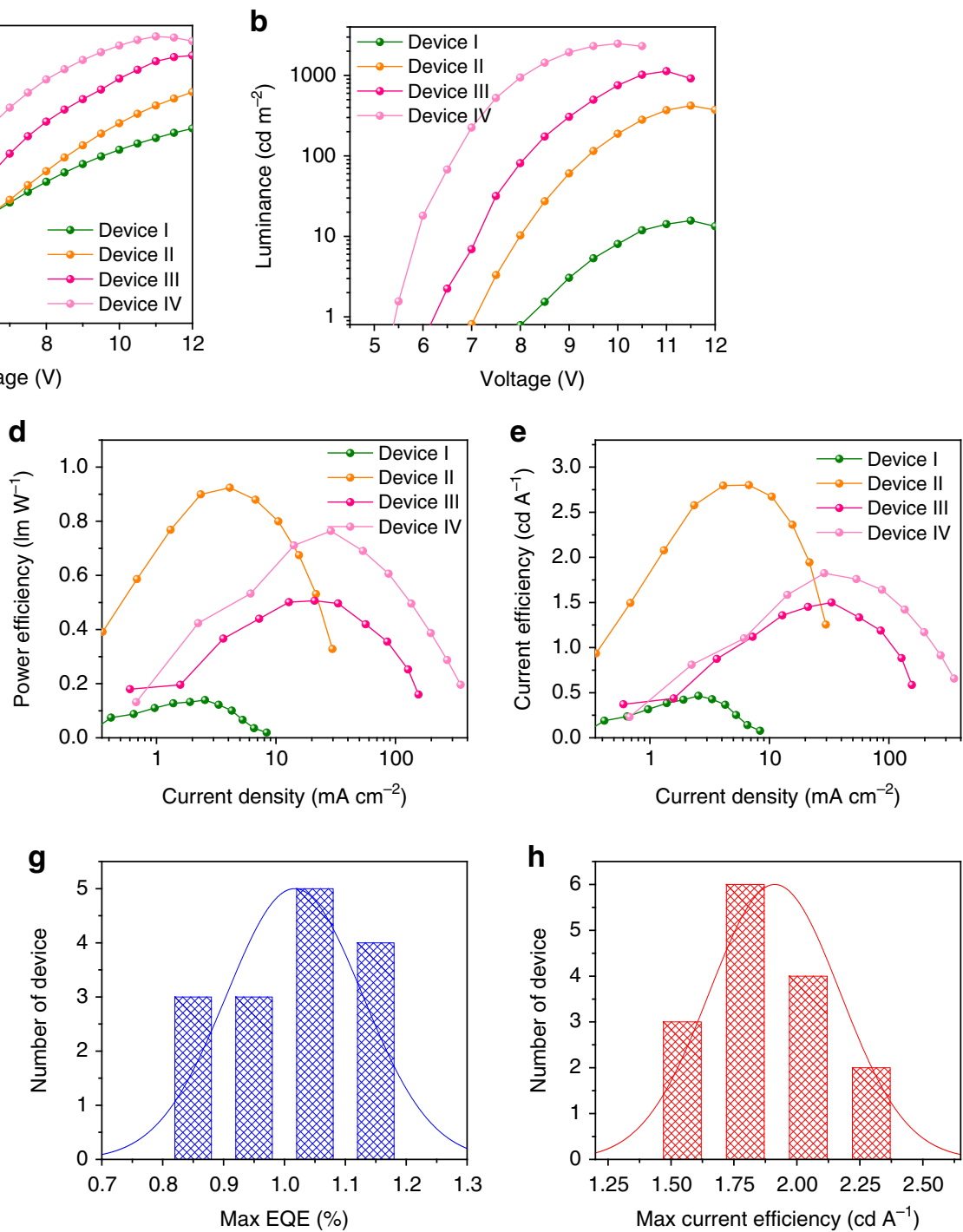

Fig. 4 EL performance of PeLEDs. a Current density-voltage, b luminance-voltage, c EQE-current density, $\mathbf{d}$ power efficiency-voltage and $\mathbf{e}$ current efficiency-voltage characteristics of PeLEDs made from perovskite films with different thickness: Device I (125 nm), Device II ( $80 \mathrm{~nm})$, Device III (60 nm), and Device IV $(40 \mathrm{~nm}) . \mathbf{f}-\mathbf{h}$ Histogram of maximum luminance, EQEs, current efficiency for 15 Devices IV from four batches. Average luminance of $1758 \mathrm{~cd} \mathrm{~m}^{-2}$ with a relative standard deviation of $22 \%$, average EQE of $1.0 \%$ with a relative standard deviation of $11 \%$ and average current efficiency of $1.9 \mathrm{~cd} \mathrm{~A}^{-1}$ with a relative standard deviation of $14 \%$

the best luminance could still be realized in a device with higher maximum current density. To characterize the reproducibility of the PeLED, we summarized the sample results such as maximum luminance, EQE, and current efficiency of Devices IV with record luminance. Figure $4 \mathrm{f}-\mathrm{h}$ shows an average luminance of $1758 \mathrm{~cd}$ $\mathrm{m}^{-2}$ with a relative standard deviation of $22 \%$, an average EQE of $1.0 \%$ with a relative standard deviation of $11 \%$ and an average current efficiency of $1.9 \mathrm{~cd} \mathrm{~A}^{-1}$ with a relative standard deviation of $14 \%$.

The color stability of the PeLEDs was tested during the device operation. As bias voltage increased from 5 to $10 \mathrm{~V}$, the EL intensity increased correspondingly, and the EL peak of $490 \mathrm{~nm}$ and full-width at half-maximum (FWHM) of $28 \mathrm{~nm}$ remained stable (Fig. 5a). When we further gradually increased bias voltage up $13 \mathrm{~V}$, the EL intensity decreased accordingly mainly due to the degradation of the perovskite materials (Supplementary Fig. 5), yet both the EL peak position and FWHM remained unchanged. The EL spectra of the PeLEDs also changed negligibly under continuous operation for $35 \mathrm{~min}$ (Fig. 5b). The stability of PeLEDs is still a big challenge, especially for blue PeLEDs, which work under higher driving voltage compared to green and red PeLEDs. The sky-blue PeLEDs lifetimes were measured to be 10 , 4 , and $0.5 \mathrm{~min}$ at initial luminance of 10,20 , and $210 \mathrm{~cd} \mathrm{~m}^{-2}$, respectively (Fig. 5c). This is consistent with the empirical scaling law $L_{0}^{n} \cdot T_{1 / 2}=C$, where $L_{0}$ is initial luminance, $T_{1 / 2}$ is LEDs halflifetime, $n$ is a scaling factor and $C$ is a constant. This equation implies that higher initial luminance will result in lower lifetime.

To investigate the effect of the additive IPABr on LEDs performance, we fabricated LEDs based on perovskite samples I, II, III, IV, and VII with the thickness of $40 \mathrm{~nm}$. The current densities of these devices decreased as the fraction of IPABr increased; meanwhile, their maximum luminance decreased from ca. 33,000 (Sample I device) to 32,500 (Sample II device), 21,000 (Sample III device), 1700 (Sample IV device) and $2 \mathrm{~cd} \mathrm{~m}^{-2}$ (Sample VII device) accordingly (Supplementary Fig. 6). This may be due to the fact that low conductive ligands blocking charge injection. The maximum EQE of Sample I and II devices are similar (ca. 2.6\%), and this value increases to $4.0 \%$ in Sample III device, and then decrease to $2.2 \%$ in Sample IV device and further to $0.05 \%$ in Sample VII device. This is consistent with the 

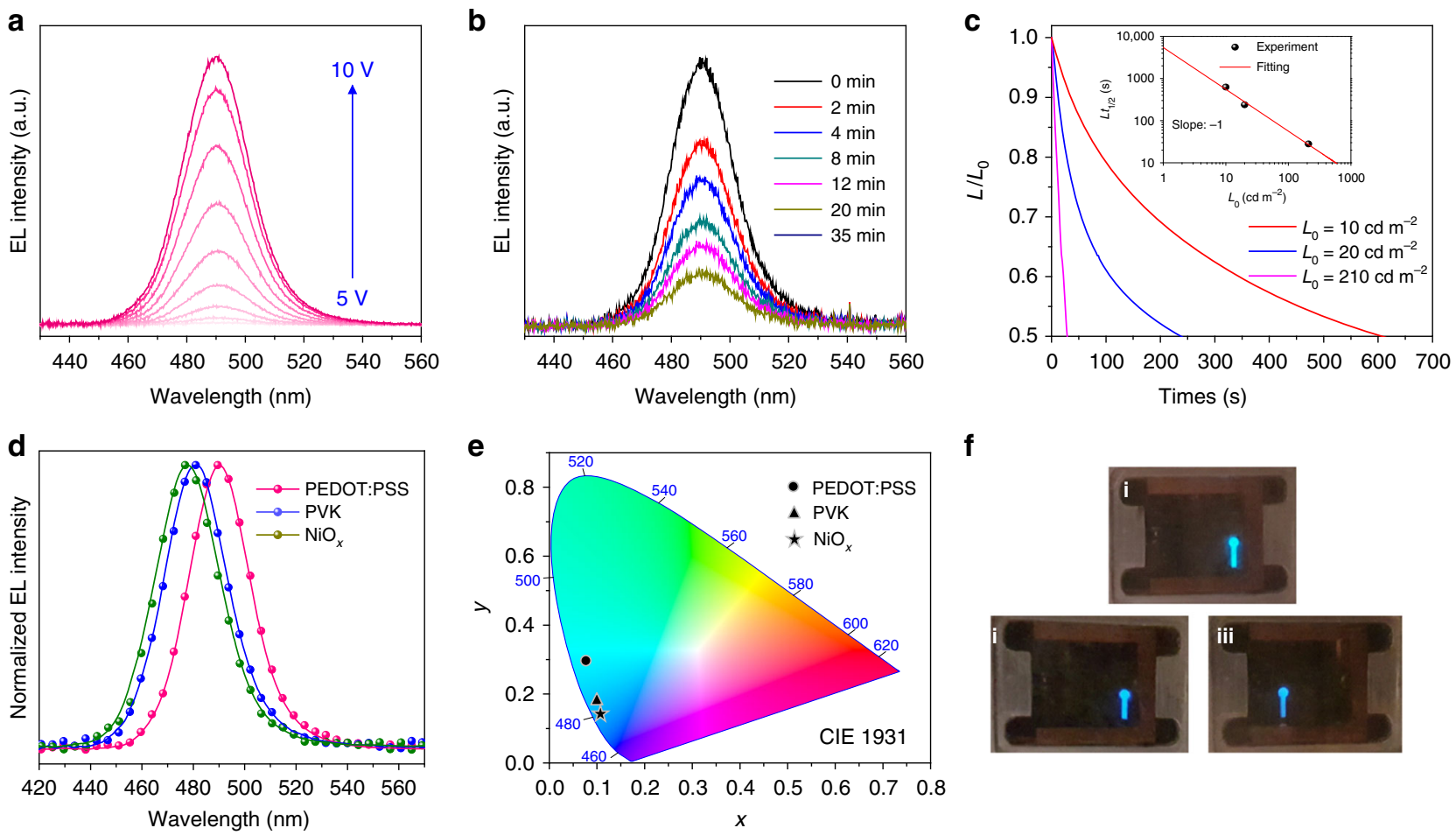

Fig. 5 EL spectra of PeLEDs. a EL spectra of PeLEDs operating under different voltage. b EL spectra of PeLED operating with various exposure times. c Lifetime measurement of the PeLEDs device at different initial luminance. The inset is estimated half-lifetime using the stretched exponential decay. $\mathbf{d}$ EL spectra and e CIE 1931 chromatic coordinates of the PeLEDs with PEDOT:PSS, PVK or NiO $\mathrm{Nas}_{x} \mathrm{HTL}$. f Digital photographs of the operating PeLEDs with PEDOT:PSS (i), PVK (ii), and $\mathrm{NiO}_{x}$ (iii) as the HTL materials, respectively

trend of PLQY. The EL signal of these devices gradually blue shifted from 513 to $474 \mathrm{~nm}$ as the fraction of IPABr increased from 0 to $60 \%$. The EL peaks also red shifted compared to their corresponding PL peaks.

The EL redshift of PEDOT:PSS-based PeLEDs may be induced by the ionic nature of PEDOT:PSS. We also therefore explored devices with neutral HTLs PVK and $\mathrm{NiO}_{x}$. Using PVK and $\mathrm{NiO}_{x}$ improved hole injection, which can be explained by their lower HOMO level compared to that of PEDOT:PSS (Fig. 3c). The threshold to achieve $1 \mathrm{~cd} \mathrm{~m}^{-2}$ decreased from $6.2 \mathrm{~V}$ (PEDOT: PSS) to $3.8 \mathrm{~V}(\mathrm{PVK})$ and $4.7 \mathrm{~V}\left(\mathrm{NiO}_{x}\right)$ (Supplementary Fig. 7). Their corresponding EL spectra are consistent with the PL spectra of the perovskite film on glass substrate, located at $481 \mathrm{~nm}$ (PVK) and $477 \mathrm{~nm}\left(\mathrm{NiO}_{x}\right)$ (Fig. 5d). These enable CIE 1931 chromatic coordinates $(0.098,0.174)$ and $(0.107,0.137)$ for purer-blue devices (Fig. 5e). Figure $5 \mathrm{f}$ shows the photographs of operating PeLEDs with PEDOT:PSS, PVK and $\mathrm{NiO}_{x}$ as the HTL material, respectively. Emission color stability was also monitored during device operation. Even though the operating voltage was increased to $8.5 \mathrm{~V}$, the EL intensity increased with the EL peak positions and FWHM remaining stable (Supplementary Fig. 8).

To identify the influence of different HTLs on the structure of the perovskite films, we first studied the PL spectra of the perovskite films fabricated on glass, $\mathrm{NiO}_{x}, \mathrm{PVK}$, and PEDOT:PSS substrates, respectively. The PL peaks of the perovskite films on glass and $\mathrm{NiO}_{x}$ are all located at $477 \mathrm{~nm}$; for PVK substrate, the PL peak slightly redshifts to $478 \mathrm{~nm}$; however, the PL peak redshifts to $488 \mathrm{~nm}$ for PEDOT:PSS substrate (Supplementary Fig. 9). UV-Vis absorption and TA spectra were used to characterize the bandgap and excitonic feature of these perovskite films (Supplementary Fig. 9). We also observed a redshift by 10 $\mathrm{nm}$ of the absorption edge of the perovskite film on PEDOT:PSS substrate. In TA spectra, three distinct bleach peaks were observed from perovskite films on glass, $\mathrm{NiO}_{x}$, and PVK substrates, which correspond to the perovskite phases with $n=$ 2,3 , and 4 . In contrast, in the perovskite film on PEDOT:PSS substrate, the perovskite phase with $n>4$ appears, as evidenced from one additional feature around $480-490 \mathrm{~nm}$. Therefore, the EL redshift of the LED device with PEDOT:PSS as HTL is attributed to the perovskite phase with large $n$ induced by PEDOT:PSS layer.

\section{Discussion}

In this study we report the synthesis of color-stable blue-emitting perovskite thin film with high PLQYs. Our approach was to deliberately select cation combinations to modulate the crystallization of quasi-2D perovskites. When IPA and PEA ligands were used simultaneously, the growth of lowest- $n$ and highest- $n$ phases was inhibited, allowing the growth of intermediate- $n$ phases to dominate instead. The resultant films display highly efficient PL and stable blue emission. Efficient and color-stable sky-blue PeLEDs have been made from these perovskite films and the devices show record high bright luminance and high EQE.

\section{Methods}

Materials and chemicals. All alkylamine bromides were purchased from Dyesol. Cesium bromide $(\mathrm{CsBr})$ and lead bromide $\left(\mathrm{PbBr}_{2}\right)$ were purchased from Alfa Aesar. DMSO, toluene, chlorobenzene, and lithium fluoride ( $\mathrm{LiF}$ ) were purchased from Sigma-Aldrich. PEDOT:PSS (AI 4083) was purchased from Heraeus. 1, 3, 5tris $(N$-phenylbenzimiazole-2-yl)benzene (TPBi) were purchased from Lumtec. All chemicals were used as received.

Perovskite film fabrication. The precursor solution was prepared by dissolving certain quantities of $\mathrm{PbBr}_{2}, \mathrm{CsBr}, \mathrm{MABr}, \mathrm{PEABr}$, and IPABr in DMSO under continuous stirring for $2 \mathrm{~h}$ at room temperature. The resulting solution was spincoated onto the substrate via a one-step process 5000 r.p.m. for 60 s. During the spin step, $200 \mu \mathrm{L}$ of toluene were poured onto the substrate. The resulting films were then annealed at $70{ }^{\circ} \mathrm{C}$ for $10 \mathrm{~min}$ to remove the residual solvent. The thickness of the perovskite film was tuned by using the precursor solution with 
different $\mathrm{Pb}$ concentrations $0.5,0.3,0.2$, and $0.15 \mathrm{M}$. Their corresponding thicknesses are around $125,80,60$, and $40 \mathrm{~nm}$, respectively.

Perovskite film characterizations. The structure of as-grown samples was characterized by using Panalytical X'Pert Pro diffractometer. UV-Vis absorption was measured by using LAMBDA $950 \mathrm{UV} / \mathrm{Vis} / \mathrm{NIR}$ spectrometer. The morphology of the perovskite films was characterized by AFM (Cypher ES SPM) in the AC mode and field-emission scanning electron microscopy (FE-SEM, JEOL JSM-7001F).

UPS spectra of the perovskite films were measured on ITO-coated glass substrate. UPS was performed in a PHI5500 Multi-Technique system using nonmonochromatized $\mathrm{He}-\mathrm{I} \alpha$ radiation $(h v=21.22 \mathrm{eV})$. All work functions and valence-band measurements were performed at a takeoff angle of $88^{\circ}$, with chamber pressure near $10^{-9}$ Torr.

PL/PLQY measurements. Steady-state PL was recorded using a Horiba Fluorolog system equipped with a single grating and a monochromatized Xe lamp was used as the excitation source. PLQY measurements were done by coupling a Quanta-Phi integrating sphere to the Horiba Fluorolog system with optical fiber bundles. Both excitation and emission spectra were collected for the two cases of the sample directly illuminated by the excitation beam path in the integrating sphere and the empty sphere itself. The monochromatized Xe lamp was used as excitation source with wavelength of $400 \mathrm{~nm}$ and power of $1 \mathrm{~mW} \mathrm{~cm}{ }^{-2}$. PL stability was measured on HR800 Raman spectrograph and $325 \mathrm{~nm}$ laser was used as excitation source.

Ultrafast transient absorption spectroscopy. The $800 \mathrm{~nm}$ output pulse laser (1 $\mathrm{KHz}$ repetition rate, $100 \mathrm{fs}$ pulse width) from a commercial Ti:Sapphire regenerative amplifier (Spectra-Physics Spitfire) is split into two paths. One beam goes through a mechanical delay stage to pump a sapphire/ $\mathrm{CaF}_{2}$ crystal to generate a light continuum to serve as the probe pulse. The second beam is sent to an optical parametric amplifier (Spectra-Physics TOPAS) to generate pump pulses $(380 \mathrm{~nm}$, $2.8 \mu \mathrm{W}$ ). The pump and probe pulses with a cross-polarization configuration are collinearly focused on samples with a beam size of 100 and $50 \mu \mathrm{m}$, respectively, by using parabolic mirrors. A mechanical chopper with a synchronized readout of a CMOS detector is used for acquisitions of probe spectra with and without pumpinduced changes, enabling calculation of a relative differential transmission. The spectra resolution is $1 \sim 2 \mathrm{~nm}$ across the detecting range.

First-principle calculations. We used ground state DFT with the Perdew-Burke -Ernzerhof (PBE) ${ }^{37}$ GGA exchange correlation functional as implemented in the Vienna ab initio simulation package (VASP) ${ }^{38}$ to perform structural optimization of the perovskites. All calculations allowed for spin polarization. We used plane wave energy cut-off of $450 \mathrm{eV}$ and Gaussian smearing $(0.2 \mathrm{eV})$ to converge the electronic problem. The Monkhorst-Pack $k$-points mesh of 900 per atom and the force convergence criterion of $0.0005 \mathrm{eV}$ per atom $\times N$ atoms in the unit cell were used as implemented in the MPRelaxSet class of the Pymatgen Python package $e^{39-42}$. The formation energy of the perovskites was calculated according to the following formula:

$$
\begin{aligned}
\mathrm{FE}= & E\left(L_{2} \mathrm{MA}_{n-1} \mathrm{~Pb}_{n} \mathrm{Br}_{3 n+1}\right)-2 E(L \mathrm{Br}) \\
& -(n-1) E(\mathrm{MABr})-n E\left(\mathrm{PbBr}_{2}\right) \\
(L= & \text { MA, PEA, IPA })
\end{aligned}
$$

PeLEDs fabrication. Thin films of PEDOT:PSS, PVK, and $\mathrm{NiO}_{x}$ were prepared using spin-coating of the precursor solution onto the prewashed patterned ITOglass substrates. PEDOT:PSS (Clevios PVP AI 4083) solution was spin-coated in air at 4000 r.p.m. for $60 \mathrm{~s}$ followed by annealing process in air at $150^{\circ} \mathrm{C}$ for $30 \mathrm{~min}$. For the deposition of $\mathrm{NiO}_{x}$ layer, $0.5 \mathrm{M}$ nickel formate dihydrate ethylene glycol solution containing 1 molar equivalents of ethylenediamine was filtered with 0.45 $\mu \mathrm{m}$ nylon filter and spin-coated at 4000 r.p.m. for $90 \mathrm{~s}$ followed by annealing process at $300{ }^{\circ} \mathrm{C}$ in air for $60 \mathrm{~min}$. After the substrates were cooled down to room temperature, $\mathrm{O}_{2}$ plasma was used to treat the $\mathrm{NiO}_{x}$ films at a power of $50 \mathrm{~W}$ for 5 $\min$. Substrates were then used for device fabrication immediately. For the deposition of PVK layer, PVK/chlorobenzene $\left(5 \mathrm{mg} \mathrm{mL}^{-1}\right)$ solution was spincoated on the ITO-glass substrate at 3000 r.p.m. for $60 \mathrm{~s}$, and annealed at $150{ }^{\circ} \mathrm{C}$ for $30 \mathrm{~min}$ in a nitrogen-filled glovebox.

Perovskite precursor solutions were spin-coated on the PEDOT:PSS film as described above. TPBi $(50 \mathrm{~nm})$ and $\mathrm{LiF} / \mathrm{Al}$ electrodes $(1 \mathrm{~nm} / 100 \mathrm{~nm})$ were deposited using a thermal evaporation system through a shadow mask under a high vacuum of less than $10^{-4} \mathrm{~Pa}$. The device active area was $6.4 \mathrm{~mm}^{2}$ as defined by the overlapping area of the ITO and $\mathrm{Al}$ electrodes.

PeLEDs characterizations. The luminance-current density-voltage characteristics were collected by using a HP4140B picoammeter. The absolute EL power spectra of the devices were collected using an integrating sphere and an Ocean Optics USB4000 spectrometer by the mounting of the devices on the wall of the integrating sphere. The EQEs were then calculated through the measured absolute power spectra and the current density. The lifetime of LEDs was measured at constant current density. In total, about 200 PeLEDs were fabricated and tested in this work.

\section{Data availability}

The data that support the findings of this study are available from the corresponding authors upon reasonable request.

Received: 15 February 2018 Accepted: 11 July 2018

Published online: 30 August 2018

\section{References}

1. Tan, H. et al. Efficient and stable solution-processed planar perovskite solar cells via contact passivation. Science 355, 722-726 (2017).

2. Liu, M., Johnston, M. B. \& Snaith, H. J. Efficient planar heterojunction perovskite solar cells by vapour deposition. Nature 501, 395-398 (2013).

3. $\mathrm{Li}, \mathrm{X}$. et al. A vacuum flash-assisted solution process for high-efficiency largearea perovskite solar cells. Science 353, 58-62 (2016).

4. Tan, Z.-K. et al. Bright light-emitting diodes based on organometal halide perovskite. Nat. Nanotechnol. 9, 687-692 (2014).

5. Yang, S. et al. Functionalization of perovskite thin films with moisture-tolerant molecules. Nat. Energy 1, 15016 (2016).

6. Cho, H. et al. Overcoming the electroluminescence efficiency limitations of perovskite light-emitting diodes. Science 350, 1222-1225 (2015).

7. Song, J. et al. Quantum dot light-emitting diodes based on inorganic perovskite cesium lead halides ( $\left.\mathrm{CsPbX}_{3}\right)$. Adv. Mater. 27, 7162-7167 (2015).

8. Yuan, M. et al. Perovskite energy funnels for efficient light-emitting diodes. Nat. Nanotechnol. 11, 872-877 (2016).

9. Xiao, Z. et al. Efficient perovskite light-emitting diodes featuring nanometresized crystallites. Nat. Photon. 11, 108-115 (2017).

10. Xing, J. et al. High-efficiency light-emitting diodes of organometal halide perovskite amorphous nanoparticles. ACS Nano 10, 6623-6630 (2016)

11. Dou, L. et al. Solution-processed hybrid perovskite photodetectors with high detectivity. Nat. Commun. 5, 5404 (2014).

12. $\mathrm{Fu}, \mathrm{Y}$. et al. Solution growth of single crystal methylammonium lead halide perovskite nanostructures for optoelectronic and photovoltaic applications. J. Am. Chem. Soc. 137, 5810-5818 (2015).

13. Xing, J. et al. Vapor phase synthesis of organometal halide perovskite nanowires for tunable room-temperature nanolasers. Nano Lett. 15, 4571-4577 (2015)

14. Ha, S.-T., Su, R., Xing, J., Zhang, Q. \& Xiong, Q. Metal halide perovskite nanomaterials: synthesis and applications. Chem. Sci. 8, 2522-2536 (2017)

15. Xing, G. et al. Low-temperature solution-processed wavelength-tunable perovskites for lasing. Nat. Mater. 13, 476-480 (2014).

16. Zhang, Q., Ha, S. T., Liu, X., Sum, T. C. \& Xiong, Q. Room-temperature nearinfrared high-Q perovskite whispering-gallery planar nanolasers. Nano Lett. 14, 5995-6001 (2014).

17. Zhang, Q. et al. High-quality whispering-gallery-mode lasing from cesium lead halide perovskite nanoplatelets. Adv. Funct. Mater. 26, 6238-6245 (2016).

18. Su, R. et al. Room-temperature polariton lasing in all-inorganic perovskite nanoplatelets. Nano Lett. 17, 3982-3988 (2017).

19. Zhang, L. et al. Ultra-bright and highly efficient inorganic based perovskite light-emitting diodes. Nat. Commun. 8, 15640 (2017).

20. Wang, N. et al. Perovskite light-emitting diodes based on solution-processed self-organized multiple quantum wells. Nat. Photon. 10, 699-704 (2016).

21. Yan, F. et al. Highly efficient visible colloidal lead-halide perovskite nanocrystal light-emitting diodes. Nano Lett. 18, 3157-3164 (2018).

22. Sadhanala, A. et al. Blue-green color tunable solution processable organolead chloride-bromide mixed halide perovskites for optoelectronic applications. Nano Lett. 15, 6095-6101 (2015).

23. Kumar, S. et al. Efficient blue electroluminescence using quantum-confined two-dimensional perovskites. ACS Nano 10, 9720-9729 (2016).

24. Li, G. et al. Highly efficient perovskite nanocrystal light-emitting diodes enabled by a universal crosslinking method. Adv. Mater. 28, 3528-3534 (2016).

25. Kumawat, $\mathrm{N}$. K. et al. Band gap tuning of $\mathrm{CH}_{3} \mathrm{NH}_{3} \mathrm{~Pb}\left(\mathrm{Br}_{1-\mathrm{x}} \mathrm{Cl}_{\mathrm{x}}\right)_{3}$ hybrid perovskite for blue electroluminescence. ACS Appl. Mater. Interfaces 7 , 13119-13124 (2015)

26. Hoke, E. T. et al. Reversible photo-induced trap formation in mixed-halide hybrid perovskites for photovoltaics. Chem. Sci. 6, 613-617 (2015).

27. Draguta, S. et al. Rationalizing the light-induced phase separation of mixed halide organic-inorganic perovskites. Nat. Commun. 8, 200 (2017).

28. Quan, L. N. et al. Tailoring the energy landscape in quasi-2D halide perovskites enables efficient green-light emission. Nano Lett. 17, 3701-3709 (2017). 
29. Wang, Q., Ren, J., Peng, X.-F., Ji, X.-X. \& Yang, X.-H. Efficient sky-blue perovskite light-emitting devices based on ethylammonium bromide induced layered perovskites. ACS Appl. Mater. Interfaces 9, 29901-29906 (2017).

30. Chen, Z. et al. High-performance color-tunable perovskite light emitting devices through structural modulation from bulk to layered film. Adv. Mater. 29, 1603157 (2017).

31. Congreve, D. N. et al. Tunable light-emitting diodes utilizing quantumconfined layered perovskite emitters. ACS Photonics 4, 476-481 (2017).

32. Cheng, L. et al. Sky-blue perovskite light-emitting diodes based on quasi-twodimensional layered perovskites. Chin. Chem. Lett. 28, 29-31 (2017).

33. Byun, J. et al. Efficient visible quasi-2D perovskite light-emitting diodes. $A d v$. Mater. 28, 7515-7520 (2016).

34. Bi, D. et al. Efficient luminescent solar cells based on tailored mixed-cation perovskites. Sci. Adv. 2, 1501170 (2016).

35. Peng, W. et al. Ultralow self-doping in two-dimensional hybrid perovskite single crystals. Nano Lett. 17, 4759-4767 (2017).

36. Liu, J., Leng, J., Wu, K., Zhang, J. \& Jin, S. Observation of internal photoinduced electron and hole separation in hybrid two-dimentional perovskite films. J. Am. Chem. Soc. 139, 1432-1435 (2017).

37. Perdew, J. P., Burke, K. \& Ernzerhof, M. Generalized gradient approximation made simple. Phys. Rev. Lett. 77, 3865-3868 (1996).

38. Kresse, G. \& Furthmüller, J. Efficient iterative schemes for ab initio totalenergy calculations using a plane-wave basis set. Phys. Rev. B 54, 11169-11186 (1996).

39. Monkhorst, H. J. \& Pack, J. D. Special points for Brillouin-zone integrations. Phys. Rev. B 13, 5188-5192 (1976).

40. Jain, A. et al. Commentary: The materials project: a materials genome approach to accelerating materials innovation. APL Mater. 1, 011002 (2013).

41. Ong, S. P. et al. Python materials genomics (pymatgen): a robust, open-source python library for materials analysis. Comput. Mater. Sci. 68, 314-319 (2013).

42. Loken, C. et al. SciNet: lessons learned from building a power-efficient top20 system and data centre. J. Phys. Conf. Ser. 256, 012026 (2010).

\section{Acknowledgements}

Q.X. acknowledges financial support from Singapore National Research Foundation via the Investigatorship Award (NRF-NRFI2015-03) and the Competitive Research Programme (NRF-CRP14-2014-03), and Singapore Ministry of Education through AcRF Tier 2 and Tier 1 grants (MOE2015-T2-1-047 and RG 113/16). E.H.S. and all co-authors from the Department of Electrical and Computer Engineering at the University of Toronto acknowledge the financial support by the US Department of the Navy, Office of Naval Research (Grant Award No: N00014-17-1-2524), by the Ontario Research FundResearch Excellence Program, and by the Natural Sciences and Engineering Research Council of Canada (NSERC). H.T. acknowledges the Netherlands Organisation for
Scientific Research (NWO) for a Rubicon grant (680-50-1511) in support of his postdoctoral research at the University of Toronto. Computations were performed on the GPC supercomputer at the SciNet HPC Consortium. SciNet is funded by the Canada Foundation for Innovation under the auspices of Compute Canada; the Government of Ontario; Ontario Research Fund-Research Excellence; and the University of Toronto.

\section{Author contributions}

E.H.S., Q.X., and Z.-H.L. supervised the project. J.X. designed the experiments, analyzed the results and wrote the paper. J.X. fabricated the perovskite film and measured the absorption, AFM and SEM assisted by L.N.Q., X.G., and H.T. L.G. assisted to measure the PL/PLQY. Y.Z. fabricated and characterized the devices. M.A. and O.V. did the firstprinciple calculations and analyzed the results. W.Z. and J.Z. performed TA measurements and analyzed the data. G.L. performed XRD measurements and analyzed the data L.N.Q., X.G., Z.Y., J.T., and O.V. discussed and commented on the manuscript.

\section{Additional information}

Supplementary Information accompanies this paper at https://doi.org/10.1038/s41467 018-05909-8

Competing interests: The authors declare no competing interests.

Reprints and permission information is available online at http://npg.nature.com/ reprintsandpermissions/

Publisher's note: Springer Nature remains neutral with regard to jurisdictional claims in published maps and institutional affiliations.

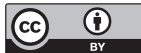

Open Access This article is licensed under a Creative Commons Attribution 4.0 International License, which permits use, sharing, adaptation, distribution and reproduction in any medium or format, as long as you give appropriate credit to the original author(s) and the source, provide a link to the Creative Commons license, and indicate if changes were made. The images or other third party material in this article are included in the article's Creative Commons license, unless indicated otherwise in a credit line to the material. If material is not included in the article's Creative Commons license and your intended use is not permitted by statutory regulation or exceeds the permitted use, you will need to obtain permission directly from the copyright holder. To view a copy of this license, visit http://creativecommons.org/ licenses/by/4.0/.

(c) The Author(s) 2018 